\title{
Thermal and mechanical design and test of the CCD mount for the WEAVE spectrograph cryostats
}

Stuart D. Bates, lain A. Steele, Chris J. Mottram, Helen E. Jermak, Niels Tromp, et al.

Stuart D. Bates, lain A. Steele, Chris J. Mottram, Helen E. Jermak, Niels Tromp, Jan Kragt, Eddy Elswijk, Johan Pragt, Remko Stuik, Robert Barnsley, Charles Monroe, Gavin Dalton, Scott Trager, Jose Alfonso Lopez Aguerri, Piercarlo Bonifacio, Antonella Vallenari, Esperanza Carrasco, Don Carlos Abrams, Kevin Middleton, "Thermal and mechanical design and test of the CCD mount for the WEAVE spectrograph cryostats," Proc. SPIE 10709, High Energy, Optical, and Infrared Detectors for Astronomy VIII, 1070912 (6 July 2018); doi: 10.1117/12.2312496

Event: SPIE Astronomical Telescopes + Instrumentation, 2018, Austin, Texas, United States 


\title{
Thermal and mechanical design and test of the CCD mount for the WEAVE spectrograph cryostats
}

\author{
Stuart D. Bates ${ }^{a}$, Iain A. Steele ${ }^{a}$, Chris J. Mottram ${ }^{a}$, Helen E Jermak ${ }^{a}$, Niels Tromp ${ }^{b}$, \\ Jan Kragt ${ }^{b}$, Eddy Elswijk ${ }^{b}$, Johan Pragt ${ }^{b}$, Remko Stuik ${ }^{c}$, Robert Barnsley ${ }^{d}$, Charles Monroe \\ Gavin Dalton ${ }^{\mathrm{d}}$, Scott Trager ${ }^{\mathrm{f}}$, Jose Alfonso Lopez Aguerri ${ }^{\mathrm{g}}$, Piercarlo Bonifacio ${ }^{\mathrm{h}}$, \\ Antonella Vallenari ${ }^{i}$, Esperanza Carrasco ${ }^{\mathrm{j}}$, Don Carlos Abrams ${ }^{\mathrm{k}}$, Kevin Middleton ${ }^{1}$ \\ aAstrophysics Research Institute, Liverpool John Moores University, IC2, Liverpool Science Park, \\ 146 Brownlow Hill, Liverpool L3 5RF, UK. \\ ${ }^{b}$ NOVA Optical- Infrared Instrumentation Group at ASTRON, Oude Hoogeveensedijk 4, 7991 PD \\ Dwingeloo, The Netherlands. \\ 'Leiden University, Leiden Observatory, P.O. Box 9513, 2300 RA Leiden, The Netherlands. \\ ${ }^{\mathrm{d}}$ Dept. of Physics, University of Oxford, Keble Road, Oxford, OX1 3RH, UK. \\ ${ }^{\mathrm{e}}$ Monroe Brothers Ltd, PO Box 12, Moreton-in-Marsh, Gloucestershire, GL56 9YX, UK. \\ ${ }_{\mathrm{f}}$ Kapteyn Institut, Rijksuniversiteit Groningen, Postbus 800, NL-9700 AV Groningen, Netherlands. \\ ${ }^{\mathrm{g}}$ Instituto de Astrofisica de Canarias, 38200 La Laguna, Tenerife, Spain. \\ ${ }^{\mathrm{h}}$ GEPI, Observatoire de Paris, Place Jules Janssen, 92195 Meudon, France. \\ i Osservatorio Astronomico di Padova, INAF, Vicolo Osservatorio 5, 35122, Padova, Italy. \\ ${ }^{\mathrm{j}}$ INAOE, Luis Enrique Erro 1, Tonantzintla, Puebla, Mexico. \\ ${ }^{\mathrm{k}}$ Isaac Newton Group, 38700 Santa Cruz de La Palma, Spain. \\ ${ }^{1}$ RALSpace, STFC Rutherford Appleton Laboratory, Harwell Oxford, OX11 0QX, UK.
}

\begin{abstract}
WEAVE is the new multi-object spectrograph for the William Herschel Telescope on La Palma. The culmination of prime focus, the large number of fibers and the wide resolution range has required a stringent optical design, which in turn demands a spectrograph with tight positional tolerances and large final focal plane. To capture this focal plane each of the two cryostats has two e2v 6k x 6k CCDs mounted as a mosaic. As well as being cooled to $150 \mathrm{~K}$ via liquid nitrogen, the positional tolerances for the sensitive areas are flatness $60 \mu \mathrm{m} \mathrm{p}-\mathrm{v}$ over the entire image area, rotation around $\mathrm{X}$ and $\mathrm{Y}$ axis \pm 50 arcmin, translation in $\mathrm{X}, \mathrm{Y}$ and $\mathrm{Z} \pm 50$ micron.

We have used a Stil confocal measuring head mounted on two Thorlab translation stages to create a X,Y mount, controlled by a Raspberry Pi that is capable of recording measurements in $\mathrm{Z}$ to better than $1 \mu \mathrm{m}$ accuracy. This is used to measure the flatness and deformation of the image area under vacuum, and when cooled to $150 \mathrm{~K}$ and the overall tip and tilt of the image plane to ensure they meet specification and are repeatable.

In addition to this measuring system, we use a Thorlabs CMOS camera with a Navitar 50mm lens to ensure each CCDs image area is within specification with regards $\mathrm{X}$ and $\mathrm{Y}$ translation.

In order to satisfy the above requirements, we designed the CCD mount to be adjustable (on initial setup), correctly constrained, isolated from liquid nitrogen boil-off vibration, and thermally insulating.
\end{abstract}

Keywords: Cryostat, CCD, Vacuum, Thermal, WEAVE

High Energy, Optical, and Infrared Detectors for Astronomy VIII, edited by Andrew D. Holland, James Beletic, Proc. of SPIE Vol. 10709, 107091Z - (c) 2018 SPIE

CCC code: $0277-786 \mathrm{X} / 18 / \$ 18 \cdot$ doi: $10.1117 / 12.2312496$ 


\section{INTRODUCTION}

WEAVE $^{1,2,3}$ is the next generation, multi-object, wide field spectrograph for the $4.2 \mathrm{~m}$ William Herschel Telescope. The project is now in the manufacture ${ }^{3}$, assembly and integration phase with first light scheduled towards the end of 2019. Light is transferred from the telescope prime focus to the dual arm spectrograph via $\sim 1000$ fibres. Wavelength coverage is $366-959 \mathrm{~nm}$, and resolution $\mathrm{R}=3,000$ to 25000 .

Each arm of the spectrograph feeds a cryostat that contains $2 \mathrm{x}$ e2 $\mathrm{v}$ CCD231-C6 detectors with $\sim 6 \mathrm{k} \mathrm{x} 6 \mathrm{k}$ pixels (Figure 1). In this paper we will show our design for the WEAVE cryostat CCD mount, the reasons for the design choices made and how the design satisfies both the mechanical and thermal requirements.

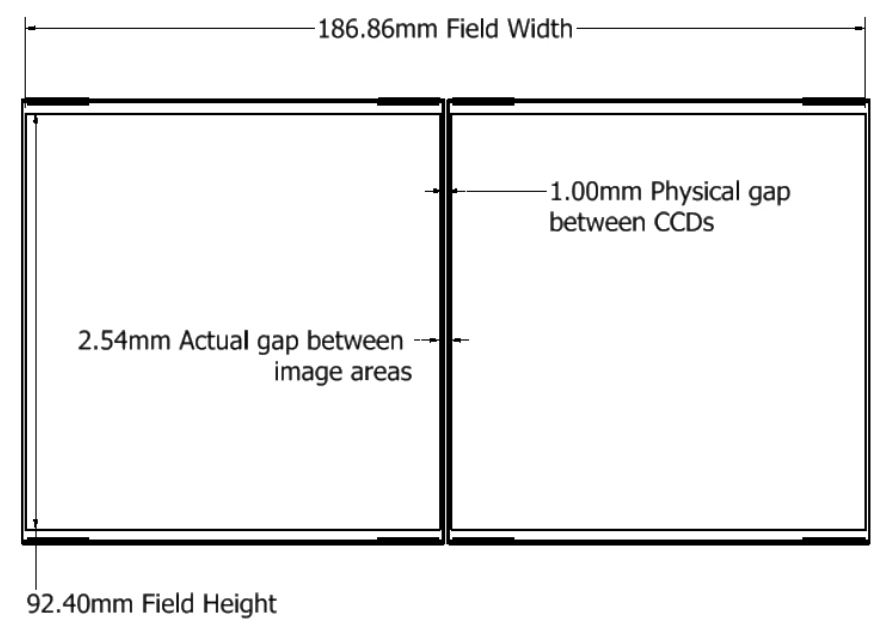

Figure 1 - Focal plane layout

The spectrograph will be situated within a clean room on one of the telescope's two Nasmyth platforms within a thermally controlled environment ("The GHRIL room"). This has dictated a tight space envelope for the spectrograph and in turn limited space for the cryostats. Figures 2 shows views of the red cryostat within its space envelope. In order to fit in this limited space envelope, the cryostat is cuboid in shape; this required extra care regarding deformation of the cryostat walls under vacuum.

The cryostat contains a $6 \mathrm{~L}$ liquid nitrogen bath (see Section 3), thermally coupled to the CCD assembly with flexible copper braid to reduce any vibration within the bath, being transferred to the CCDs. Figures 3 and 4 show the cryostat components.

The following positional constraints apply to the mounting of both CCDs in each cryostat:

1. The image plane is to be made up of two $6 \mathrm{k} x 6 \mathrm{k}$ CCDs, with a physical separation of $1 \mathrm{~mm}$ along one edge

2. The entire surface of this image plane needs to fall between two theoretical, parallel planes, $60 \mu \mathrm{m}$ apart.

3. The distance between the outside surface of the cryostat window and the last surface of the camera lens assembly is $\sim 5 \mathrm{~mm}$.

4. The window thickness is set at $15 \mathrm{~mm}$

5. The center of this image plane needs to be $3.287 \mathrm{~mm} \pm 50 \mu \mathrm{m}$ for the red cryostat and $3.532 \mathrm{~mm} \pm 50 \mu \mathrm{m}$ from the inside surface of the blue cryostat window, $(\mathrm{Z}$ axis, piston)

6. Although parallel to the window in the spatial direction $( \pm 1 \mathrm{arcmin})$, this image plane needs to be at an angle of $+0.266 \mathrm{deg} \pm 1$ arcmin (red) and $-0.278 \mathrm{deg} \pm 1$ arcmin (blue), with the inside surface of the cryostat window in the spectral direction. 
7. This image plane also needs to be held in position to within $\pm 50 \mu \mathrm{m}$ in translation, ( $\mathrm{X}$ and $\mathrm{Y}$ axis).

Items 3, 4 and 5 of the above list have made the mounting of the cryostat window challenging. No material can be fitted beyond the outside surface of the window, (which would normally be required to apply a given preload to any sealing $\mathrm{O}$ ring or metal seal), or between the CCDs and the inside surface of the window. In addition, as the thickness of the window is set at $15 \mathrm{~mm}$, the $\mathrm{X}$ and $\mathrm{Y}$ dimensions of the window need to as small as possible to maintain the mechanical integrity of the window itself, and to reduce deformation of the window to a minimum. This has also affected the CCD Mount design.

A cryostat with an integrated internal brace as part of the overall body was designed to reduce deformation of this front face of the assembly, therefore reducing any deformation transferred into the CCD assembly itself.

The CCD assembly as a whole has to fulfill a number of purposes:

- Be stiff enough to maintain a set position for the image plane in all degrees of freedom

- Have a level of adjustment to allow a given tilt of the image plane to be set to the required level of accuracy on initial assembly

- Be able to maintain that position when the vacuum is applied

- To be constrained sufficiently so as to not deform the image area when the CCDs are cooled to 150K.

- Allow the image area access to be positioned at the required distance from the inside of the cryostat window $(\sim 3.25 \mathrm{~mm})$.

- Be thermally insulating

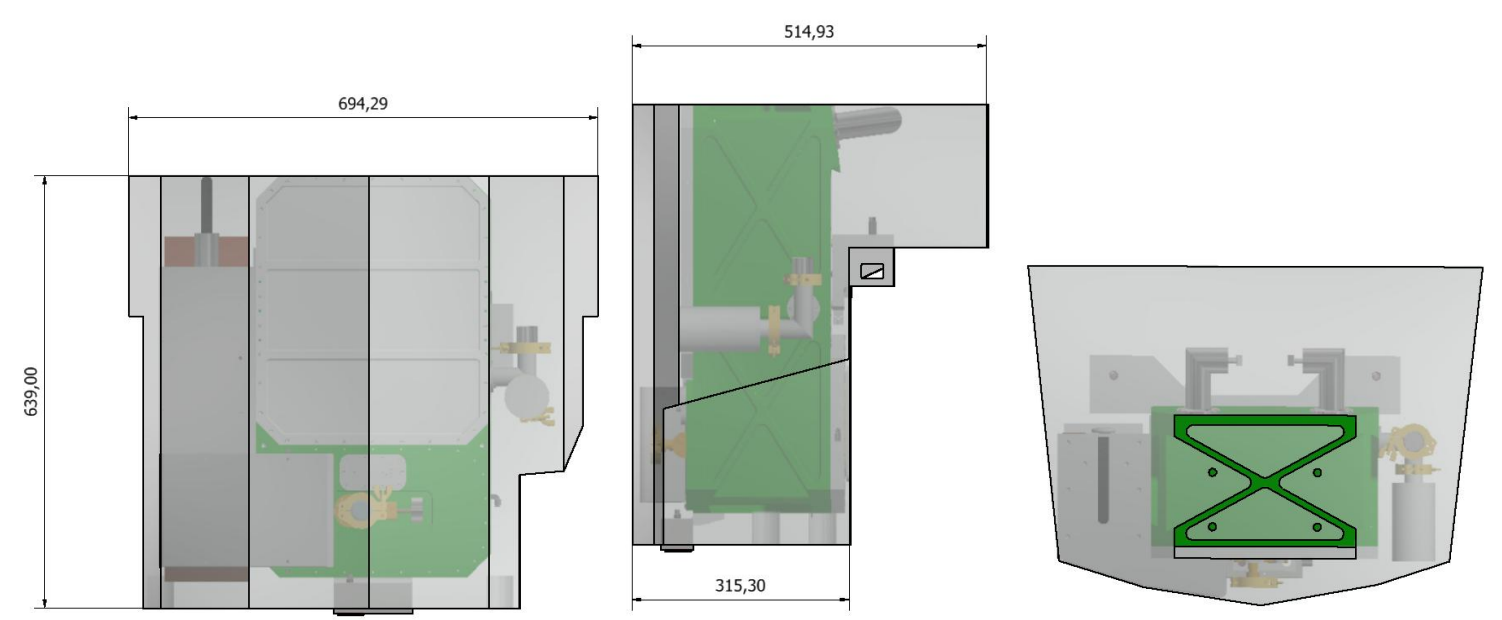

Figure 2 Rear, side and top views of cryostat, with space envelope shown as wire-frame 


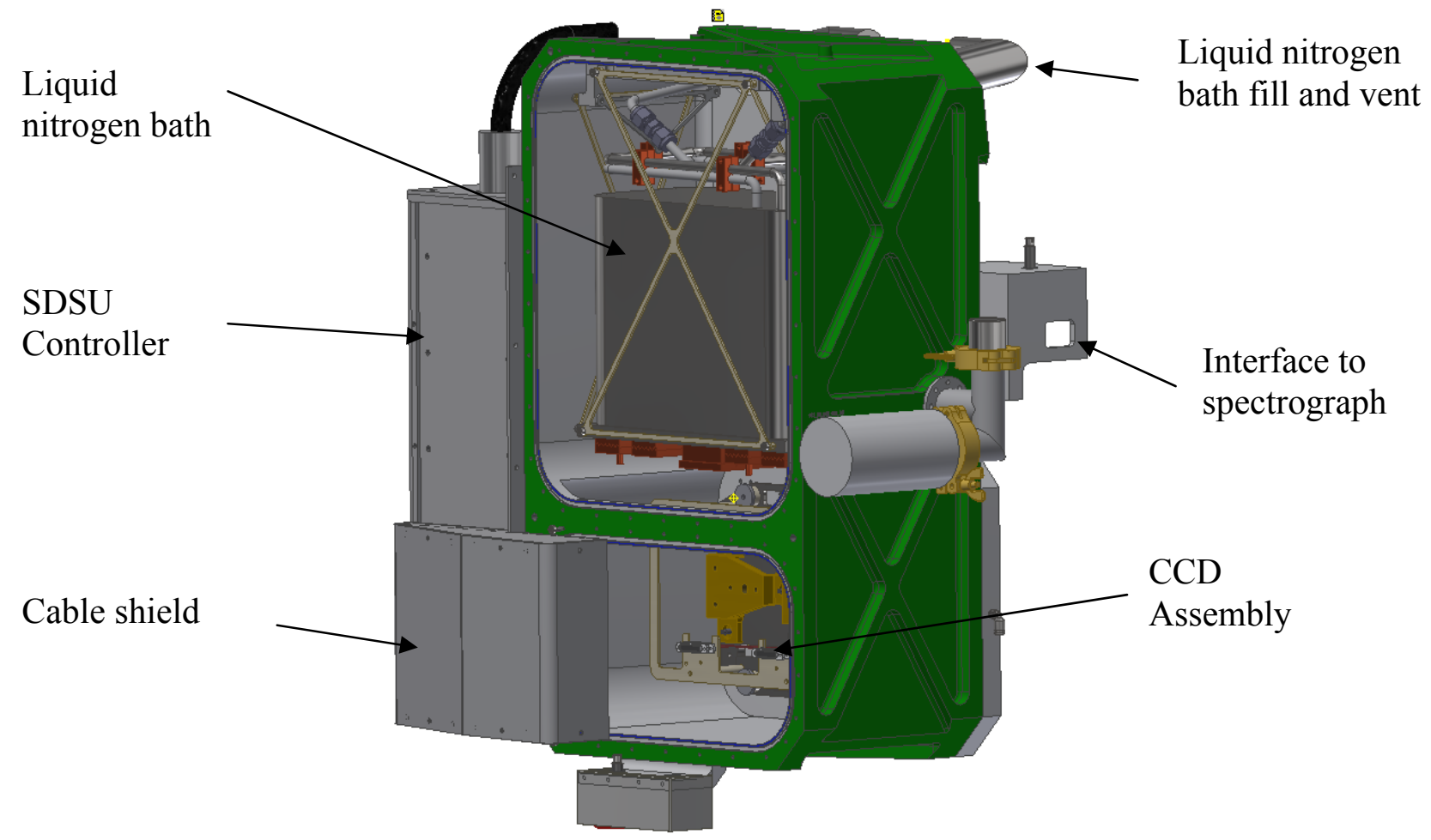

Figure 3 3D Model showing rear of the red cryostat

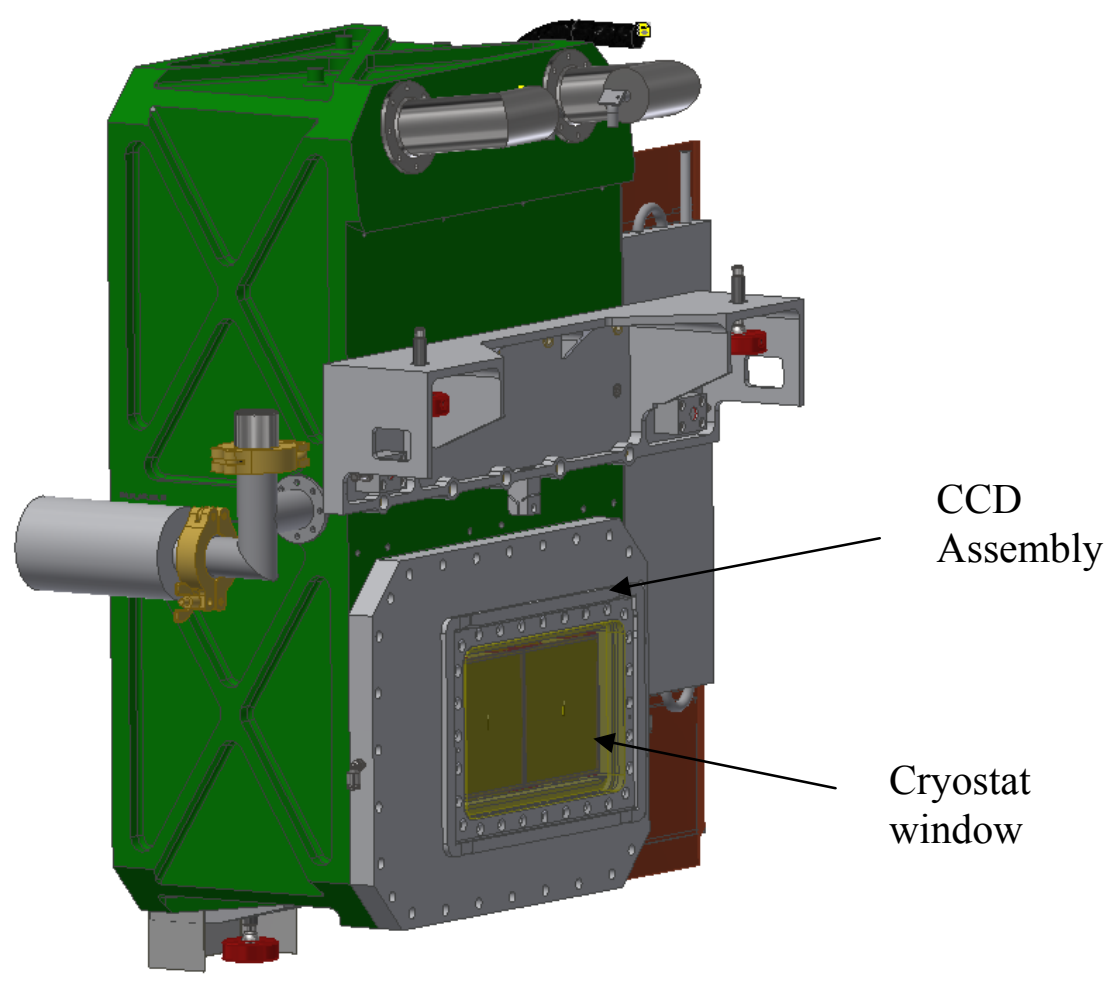

Figure 4 3D model showing front of red cryostat 


\section{MECHANICAL DESIGN}

We developed a CCD assembly that referenced all key optical components to one structural item, the CCD Reference Plate. This contains the window support features and has the rest of the CCD assembly fastened to it to maintain a fixed relationship between the CCD image areas and the cryostat window.

In order to maintain co-planar alignment of the two CCDs in each cryostat, the bulk of the CCD assembly is a stainless steel mounting plate, ground to a flatness of better than $5 \mu \mathrm{m}$, (Figure 5).

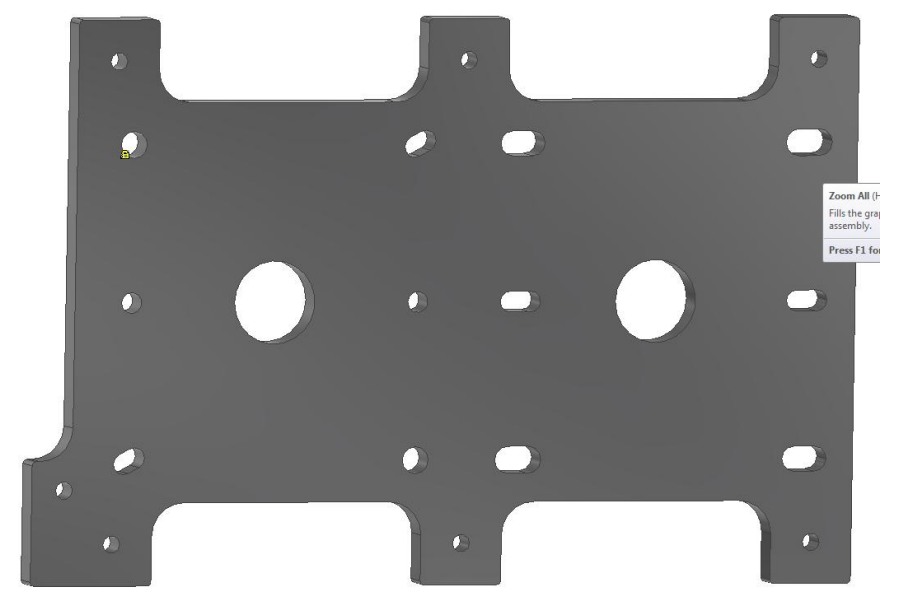

Figure 5 CCD mounting plate

The measured results provided by e $2 \mathrm{v}$ for each CCD show that all points measured on each CCD image area fall within the given flatness specification, therefore allowing us a resulting tolerance of $\pm 30 \mu \mathrm{m}$.

This CCD mounting plate is supported along the top and bottom edges with three mounting positons to limit any deformation transferred into the plate itself (Figure 6). These structures are attached to the CCD Reference Plate via a length of stud and compression springs to allow for thermal contraction when cooling and adjustment of the image area on assembly.

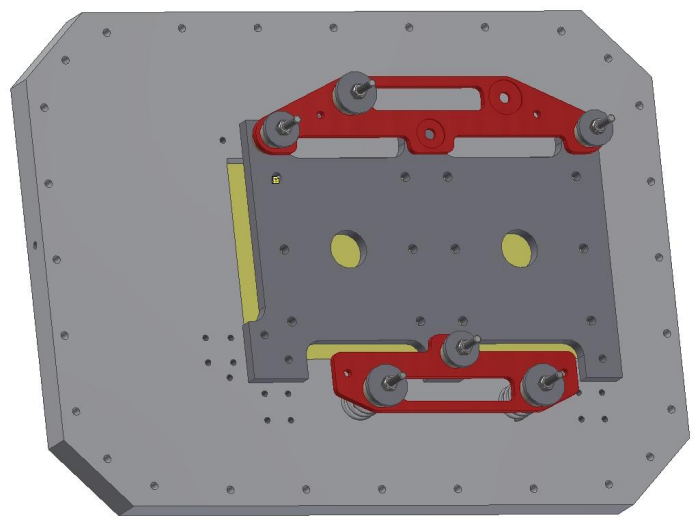

Figure 6 CCD mounting plate support

The upper and lower parts (mount brackets) shown in Fig 6 are produced from Invar, this reduces any contraction or expansion due to temperature changes due the Invar's low CTE. In addition this acts as additional thermal insulation due to Invar's lower thermal conductance. The stainless steel compression springs (shown in Figure 7) also act as effective insulation due to their comparatively long thermal path and low cross sectional area. The stainless steel studs act as a thermal short around these thermal paths. Tufnol 10G40 columns and Invar washers have been used to both extend the studs length and then insulate the studs from the mount brackets. 
Figure 7 Cross sectional view of mounting bracket support

Figure 7 shows a cross sectional view of this support arrangement. The stud's isolation from the mounting bracket is achieved by recessing the Tufnol columns and the compression springs into the mounting bracket, and allowing additional clearance around the stud as it passes through the mounting bracket. In the background of Figure 7, the support from the mounting bracket to the CCD mounting plate is also shown. This has the Tufnol columns on both sides of the plate for further insulation and support.

To eliminate any non-repeatable movement in $\mathrm{X}$ and $\mathrm{Y}$, and to allow for adjustment, lateral adjustment features have been produced and fitted (Figure 8). These have a reduced area footprint and only point contact for positioning to reduce thermal transfer.

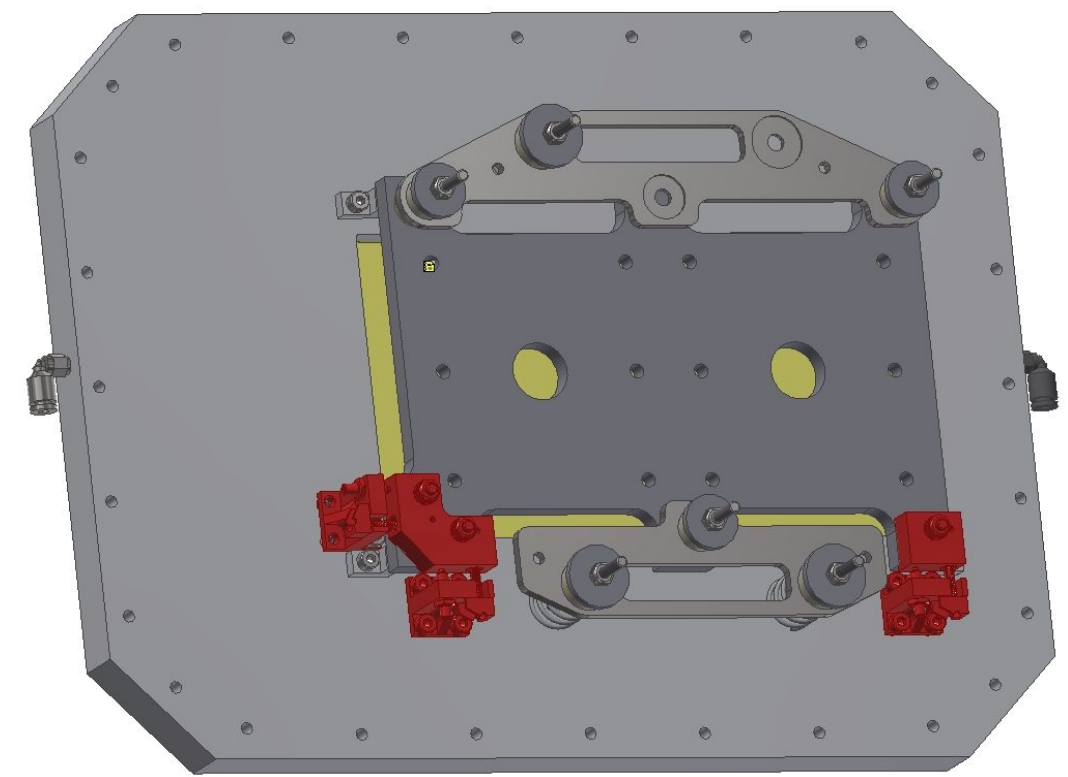

Figure 8 Image showing lateral adjustment mechanisms

There was concern that due to the close proximity of the CCD image areas to the inside surface of the cryostat window, the two may come into contact during initial set up. To mitigate this, stoppers (shown in Figure 9) were produced and fitted under the four corners of the CCD mount plate. These can also be used to bring the CCD mount plate close to the desired position by using slip gauges between each stopper and the CCD mount plate on initial assembly. 
Heat transfer is not a concern, as they will not contact the CCD mount plate when the position is adjusted correctly.

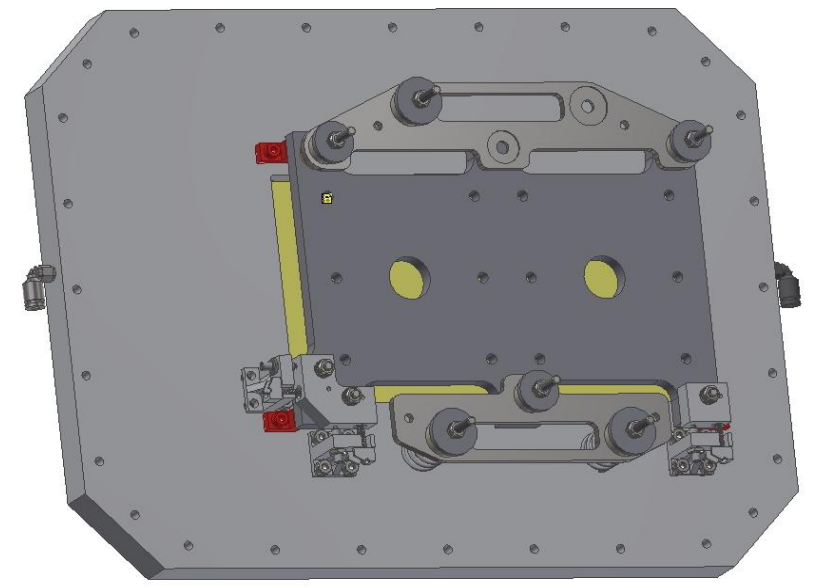

Figure 9 Image showing 'Stoppers' to protect the CCDs from contacting the cryostat window

Finally heat is transferred from the CCD mounting plate and the CCDs via copper spiders shown in Figure 10. These are mounted over the CCD support posts creating the shortest possibly path through the CCD mounting plate. These have been gold plated to prevent tarnishing, and so in turn reduce emissivity.

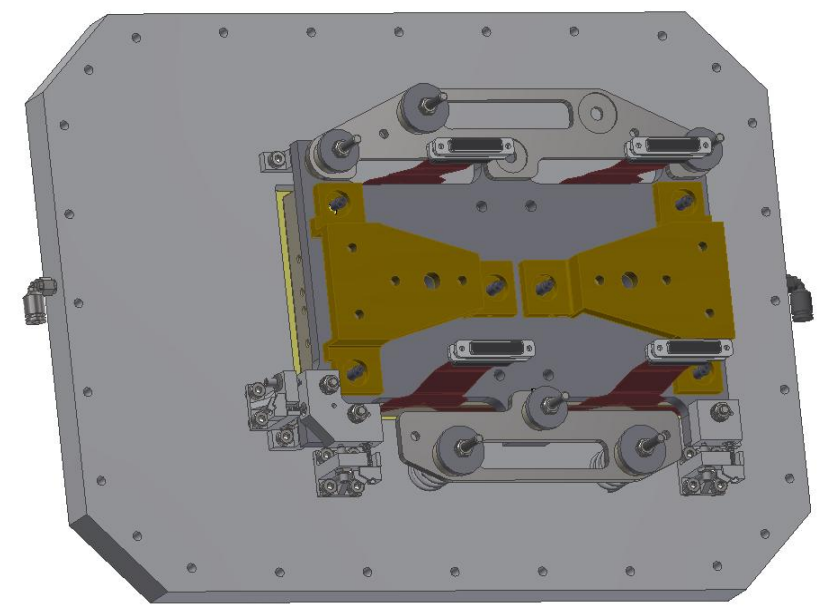

Figure 10 Complete CCD Assembly with gold plated cold spiders

\section{THERMAL ANALYSIS}

In modelling the heat input to the $\mathrm{CCD}$, we consider both conductance via the mounting structure and radiation from the cryostat body and window.

\subsection{Conductive Heat Load.}

We have simplified the calculations by ignoring the CCD mount brackets and the invar washers. Adding these items will improve thermal insulation. We calculate the head load (q) for each component via the standard formulation $\mathrm{q}=\underline{\mathrm{K} \quad \mathrm{A} \quad \Delta \mathrm{T}}$ 
where $\mathrm{q}=$ Heat load $(\mathrm{W}), \mathrm{K}=$ Thermal conductivity $\mathrm{W} /(\mathrm{m} \cdot \mathrm{K}), \mathrm{A}=$ Cross sectional Area $\left(\mathrm{m}^{2}\right), \Delta \mathrm{T}=$ Temperature Difference $(\mathrm{K})$ and $\mathrm{L}=$ Length $(\mathrm{m})$. The heat loads were then combined (Figure 11) to calculate the total head load per $\mathrm{CCD}$ via conduction through the mounting of $1.26 \mathrm{~W}$. In addition $1.51 \mathrm{~W}$ of heat load is anticipated via the CCD wiring loom.

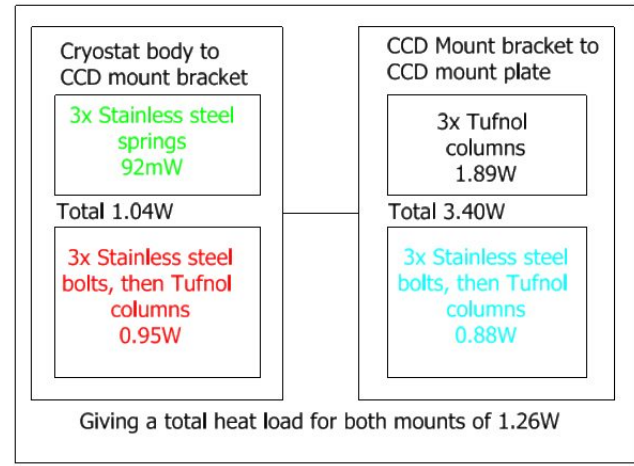

Figure 11 Representation of thermal paths in CCD mount assembly

\subsection{Radiative Head Load}

The majority of radiative heat load is through the cryostat window. We calculated this using the two surface grey body heat transfer approximation

$\dot{Q}=\frac{\sigma\left(T_{1}^{4}-T_{2}^{4}\right)}{\frac{1-\epsilon_{1}}{A_{1} \epsilon_{1}}+\frac{1}{A_{1} F_{1 \rightarrow 2}}+\frac{1-\epsilon_{2}}{A_{2} \epsilon_{2}}}$

where $\mathrm{F}_{\mathrm{i}-\mathrm{j}}$ defines the fraction of radiation exiting surface $\mathrm{i}$ that impinges on surface $\mathrm{j}, \varepsilon_{\mathrm{i}}$ is the emissivity of surface $\mathrm{i}, A_{\mathrm{i}}$ is the area of surface $\mathrm{i}, T_{\mathrm{i}}$ is the temperature of surface $\mathrm{i}$, and $\sigma$ is the Stefan-Boltzmann constant. We used shape factors from Ref [4] and emissivities from Ref [5]. The resulting heat load through the cryostat window to the CCDs 6.32W. It can be seen that this exceeds the conductive load.

\subsection{Implications for design}

Combining the conductive and radiative heat loads we arrived at a value of $\sim 10 \mathrm{~W}$. This value was used to size the number and cross section of the copper braids between the LN2 bath and the CCD mount and in modelling the LN2 hold time (specified at 15 hours) and achieved CCD temperature (150K). The resulting bath specifications are a capacity of $6 \mathrm{~L}$ for a maxium permissible heat load of $17.8 \mathrm{~W}$.

\section{EXPERIMENTAL PROCEDURES}

In order to verify the CCD mounting met requirements, a number of experiments were carried out as described in this Section. The requirements to be verified were:

- The correct image plane position in $\mathrm{Z}$ (piston)

- The correct image plane rotation around Y (spectral direction, tilt)

- No image plane rotation around X (spacial direction, tip)

- Image area flatness is within specification including any deformation of flatness through thermal change

- Stability of position whilst at temperature

- Repeatability of image plane in translation and rotation around X, Y and Z, when thermally cycled 
- Operating temperature can be achieved and held for specified time between liquid nitrogen fills

- Image position stability with regards vibration from liquid nitrogen boiling off

\subsection{Verifying position and stability in $\mathrm{Z}$, tip and tilt}

All requirements regarding the flatness of image area, position in Z, and tip and tilt were measured using a Stil Initial 12, chromatic confocal measuring head, mounted on two Thorlab LTS300/M translation stages to create a X,Y mount. (Figure 12). This is controlled with a Raspberry Pi and is capable of recording measurements in $\mathrm{Z}$ to an accuracy of $600 \mathrm{~nm}$.

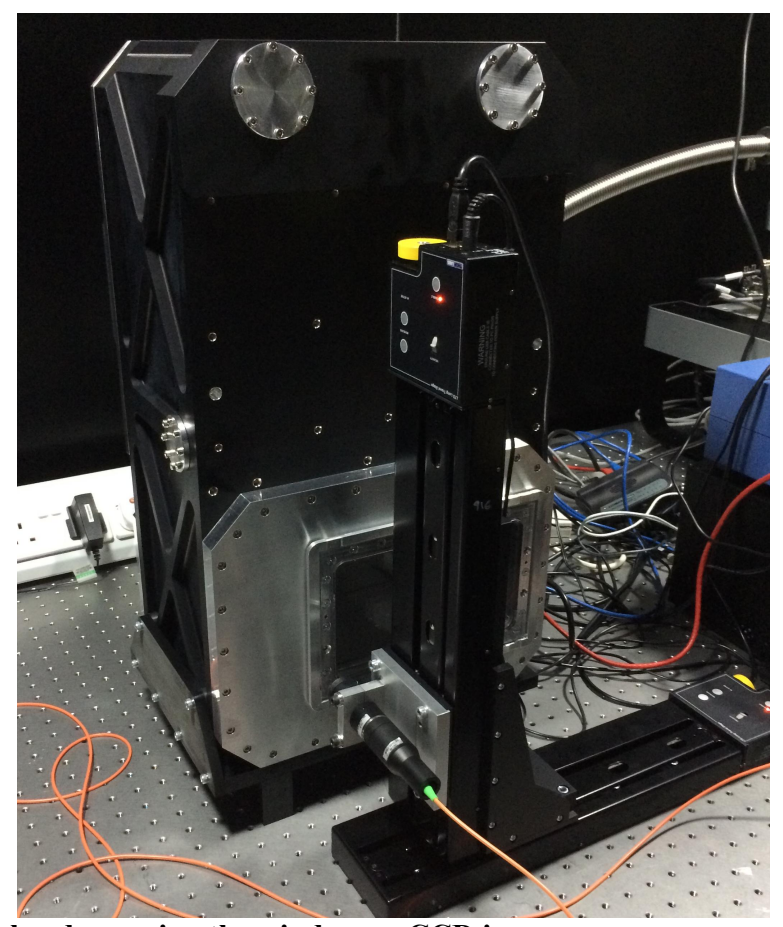

Figure 12 Confocal measuring head scanning the window or CCD image areas

The initial set up was with the confocal measuring head in a static position in the middle of the image area; this position was then set to the required depth. Then the measuring head was moved to one of the ends and the tip and tilt adjusted to the correct value. Once completed the entire window was scanned, taking a depth measurement every $5 \mathrm{~mm}$ in $\mathrm{X}$ and $\mathrm{Y}$. The Stil confocal measuring head operates by detecting the most reflective surface in its measuring range. If set correctly in Z, it will detect the CCD image area (as the most reflective, being gloss black), but then when measuring off the edges of the CCDs, it picks up the inside surface of the cryostat window. This useful feature allows both the CCD image areas and the window overall flat to be measured in the same run, producing accurate, relative results.

The data collected is split into window measurements and CCD image area measurements, and a surface plot generated for each. The window flat is subtracted from this image area surface to show the relative tip and tilt of the image areas. This procedure was followed without a vacuum applied, with a vacuum, and again when at operating temperature to show stability of image area. The cryostat was allowed to warm up to ambient temperatures, then cooled, and the procedure carried out again to check repeatability.

\subsection{Verifying lateral stability}

To confirm the CCD image areas in $\mathrm{X}$ and $\mathrm{Y}$ (translation) the entire image area was imaged using a Thorlabs CMOS camera and Navitar 50mm lens. 


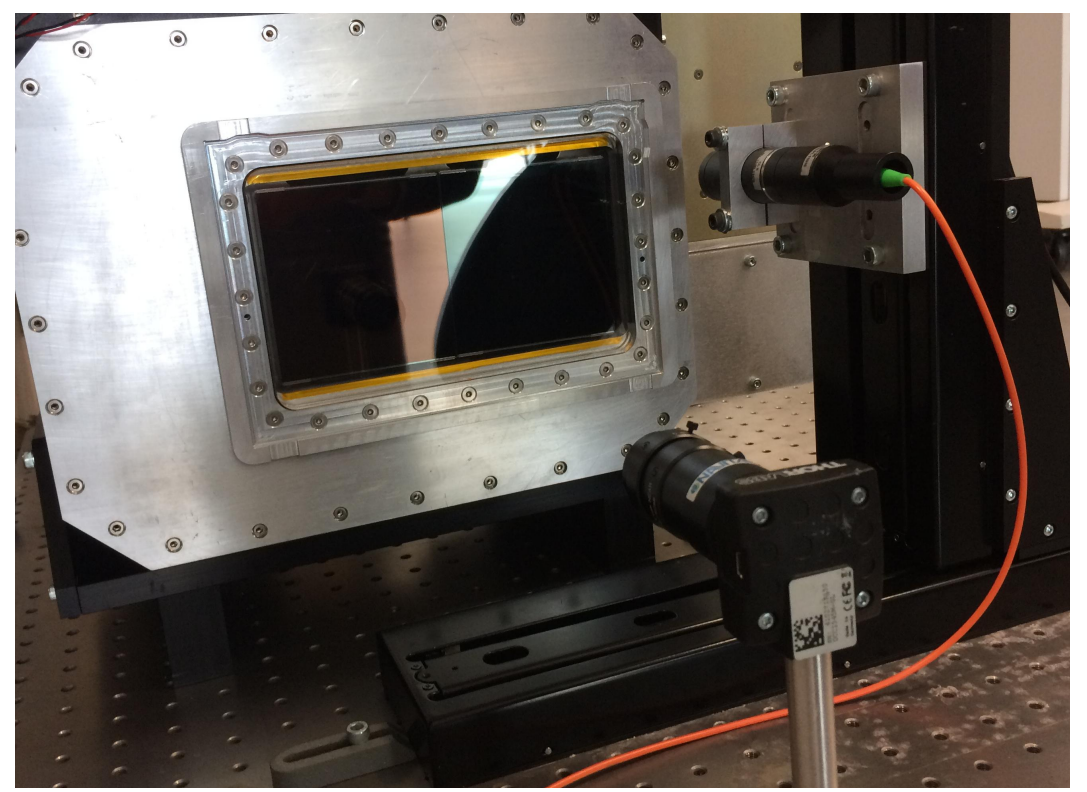

Figure 13 Image of confocal measuring system (right) and imaging camera (to bottom)

Figure 13 shows the camera with which images are taken periodically through the vacuum and cooling cycles, then cross correlated to show shifts in $\mathrm{X}$ and $\mathrm{Y}$. The cryostat is allowed to warm up to ambient and the process is repeated.

\subsection{Verify operating temperature for CCDs and liquid nitrogen hold time}

The CCDs need to be operated at $150 \mathrm{~K}$. This is hard to verify as there is no means by which to attach a temperature directly to the light sensitive areas. We monitored the CCD mount temperature via a PT100 sensor, read by the CCD controller and diode type sensors fitted to the liquid nitrogen bath base, the liquid nitrogen fill port, vent port and cold spiders, read by an Arduino. This latter system is a 'service mode' system, i.e. it is not utilized in normal operations. To prove the sensitive areas of the CCDs are sufficiently cooled, we measure the dark current via dark images.

\subsection{Image area flatness}

\section{EXPERIMENTAL Results}

In Figures 14 and 15 we show the comparison for each CCD in the red cryostat, showing flatness at ambient temperature and when at operating temperature. 


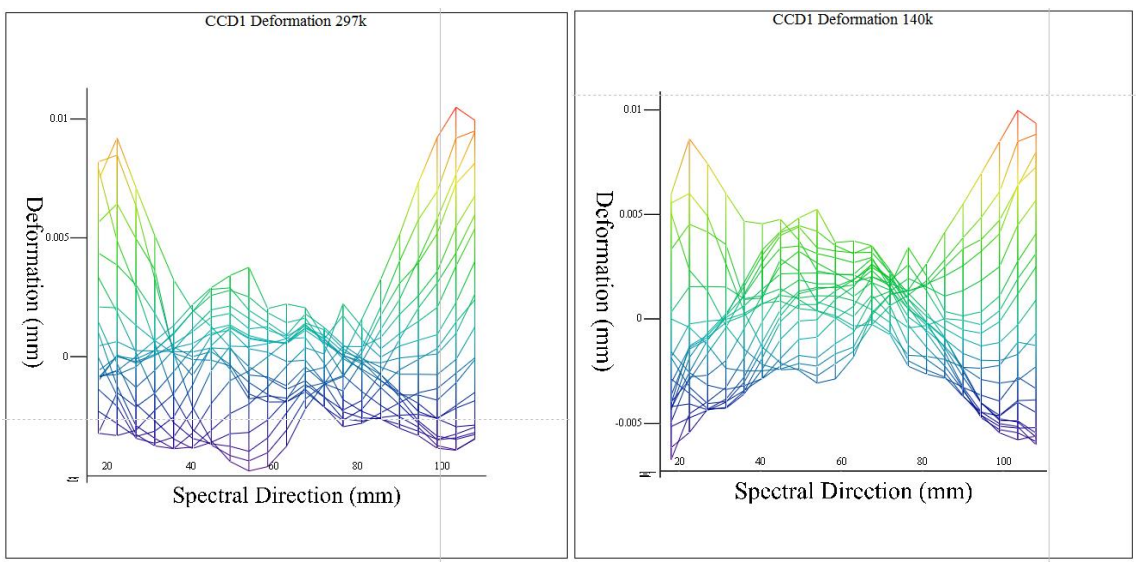

Figure 14 CCD1 Deformation at ambient and operating temperature
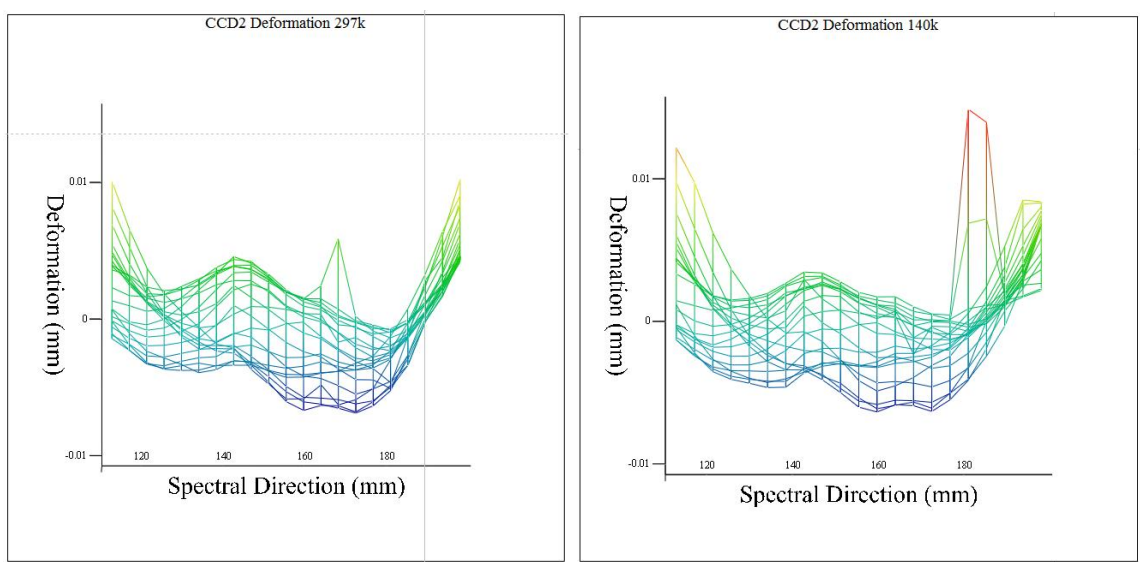

Figure 15 CCD2 Deformation at ambient and operating temperature

The surface plots show similar deformation whether the CCDs are at ambient or operating temperature. This indicates the CCDs are not being subjected to any significant internal stresses due to the difference in thermal expansion of CCD substrate and the CCD mounting plate, and the surface flatness remains well within the requirement of $\pm 30 \mu \mathrm{m}$

\subsection{Image area in $Z$, tip and tilt}

Figures 16-20 show the CCD image areas as measured at given stages throughout the assembly and set up procedure. These have been derived by:

1.Measuring the window position and deriving a flat

2.Measuring the CCD image area and deriving a flat

3.Subtracting the CCD image area flat from the CCD image area raw to give the CCD deformation in isolation

4.Subtracting the CCD image area flat from the window flat, then adding the CCD deformation onto the result

This shows the deformed CCD image area, still falls between the upper and lower tolerances for image plane position. Figures 16-20 show the image area in colour as viewed from above, with the specified limits in black 

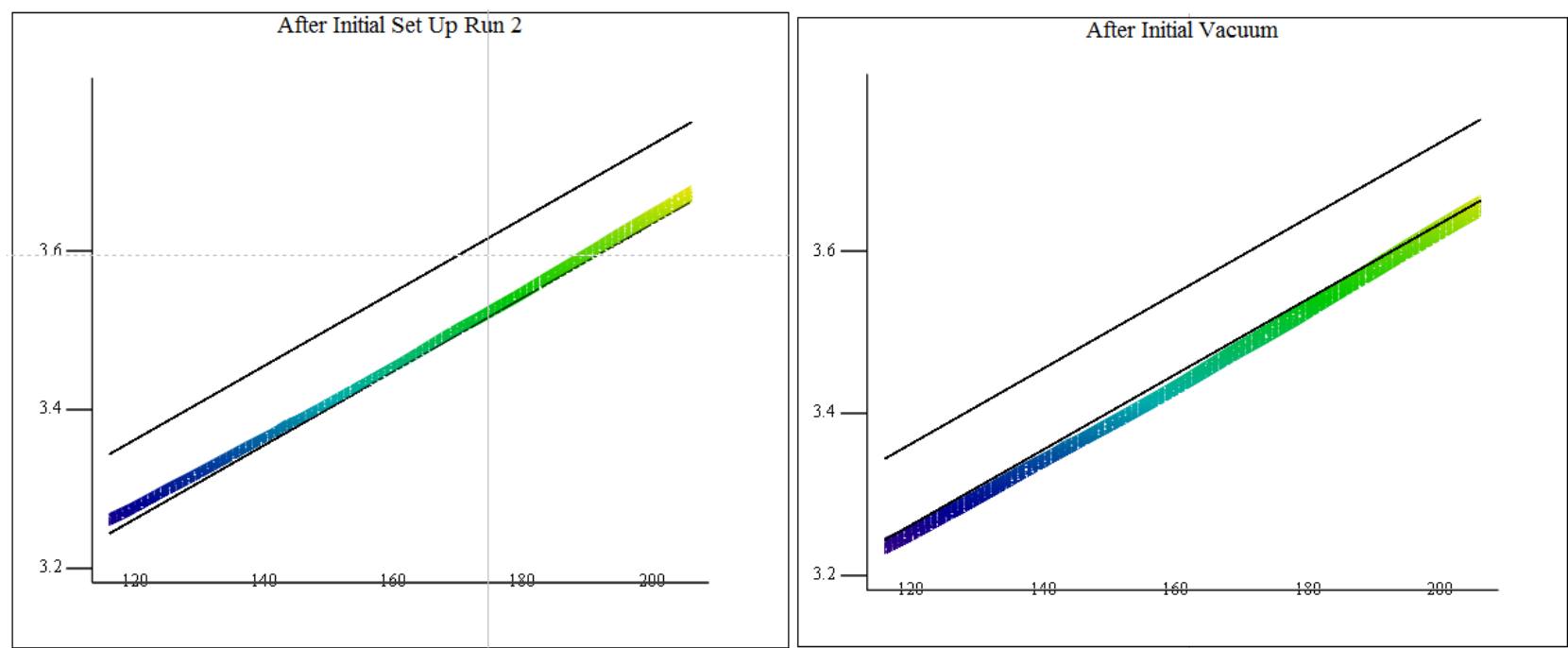

Figure 16 CCD image area after intial set up, units are mm. Figure 17 CCD image area after initial vacuum, units are mm

After an initial set up setting the image area towards the lower tolerance, applying the vacuum sends the image area out of specification as seen in figure 17 .

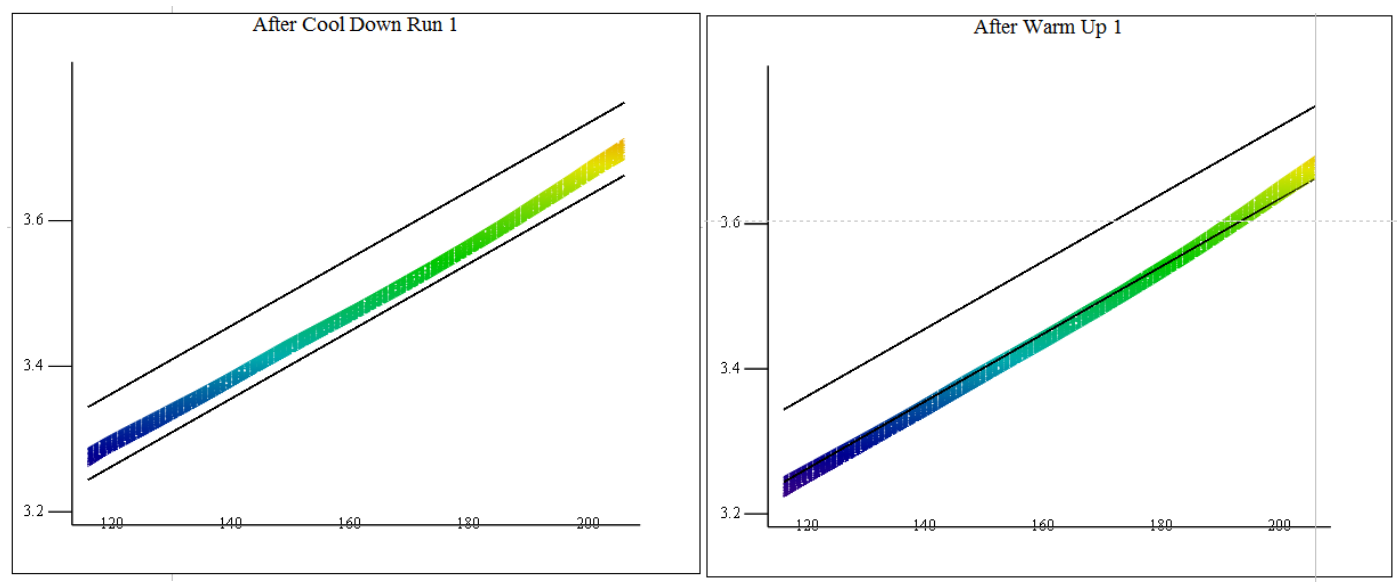

Figure 18 Image after initial cool down, units are in $\mathbf{m m} \quad$ Figure 19 Image area after first warm up, units are in $\mathrm{mm}$

Once at operating temperature, the image area moved more within specification (Figure 18). When warmed up, it returns to outside specification (Figure 19) 


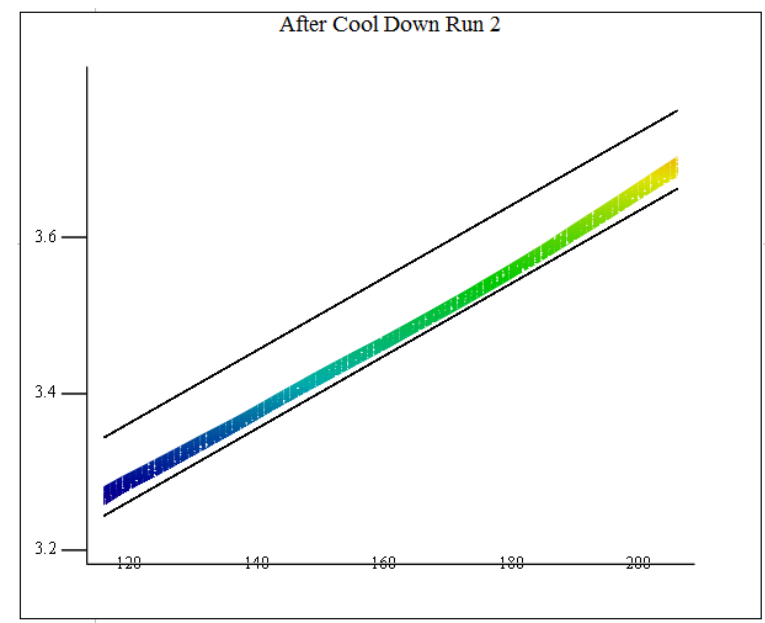

Figure 20 Image area after second cool down, units are in $\mathbf{m m}$

When cooled again (Figure 20), the image area returns to within specification. Showing that all points measured fall on a plane between 3.258 and $3.703 \mathrm{~mm}$, within the $\pm 30 \mu \mathrm{m}$ flatness, the overall tilt of $+0.266 \mathrm{deg} \pm 1$ arcmin and the $\pm 50 \mu \mathrm{m}$ specification in $\mathrm{Z}$ (or piston).

\subsection{Vibration from liquid nitrogen boil off}

The confocal measuring head was set up on one point on the CCD image area and left to generate a measurement every second. The results of this analysis are shown in Figure 21. This shows any vibration detected to be $0.12 \mu \mathrm{m}$. It also shows a $6 \mu \mathrm{m}$ range of movement over the entire period. This is assumed to be due to the ambient temperature within the laboratory, (we have seen similar results when measuring fixed components) and is well within the $\pm 50 \mu \mathrm{m}$ tolerance for the image plane in $\mathrm{Z}$. The sudden movement at the end of the graph is assumed to be the laboratory's air con starting in the morning.

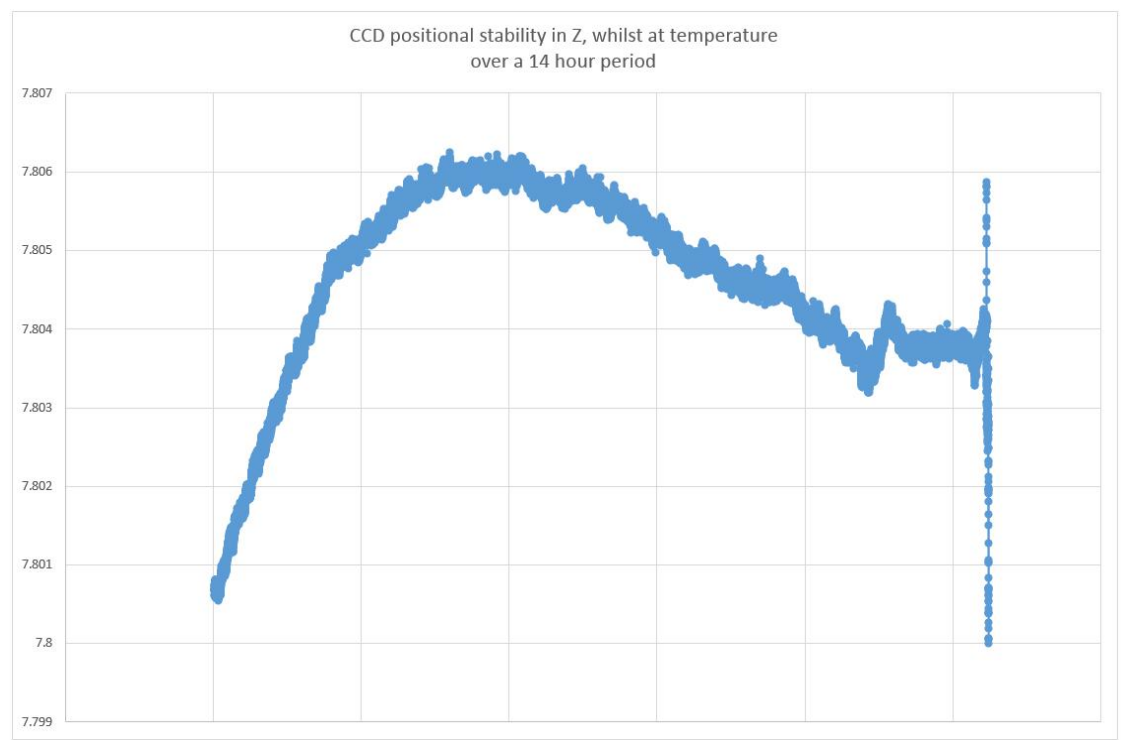

Figure 21 One point on CCD image area over a 14 hour period, the $X$ axis is time, over a 14 hour period and the $Y$ axis is CCD position in $\mathrm{mm}$. 


\subsection{Operating temperature and liquid nitrogen hold time}

Figure 22 shows the liquid nitrogen hold time (for a full bath of liquid nitrogen) and the temperature as measured at the CCD mount. This shows the hold time was from when the CCD mount plate reached operating temperature (in this case $127 \mathrm{~K}$ ) at 20:20 hrs 07/08/2017 to when the liquid nitrogen bath started to warm up at 17:44 hrs 08/08/2017 (note there is a small read error on the temperature sensors, showing the liquid nitrogen bath to be at $69 \mathrm{~K}$ instead of $77 \mathrm{~K}$ ).

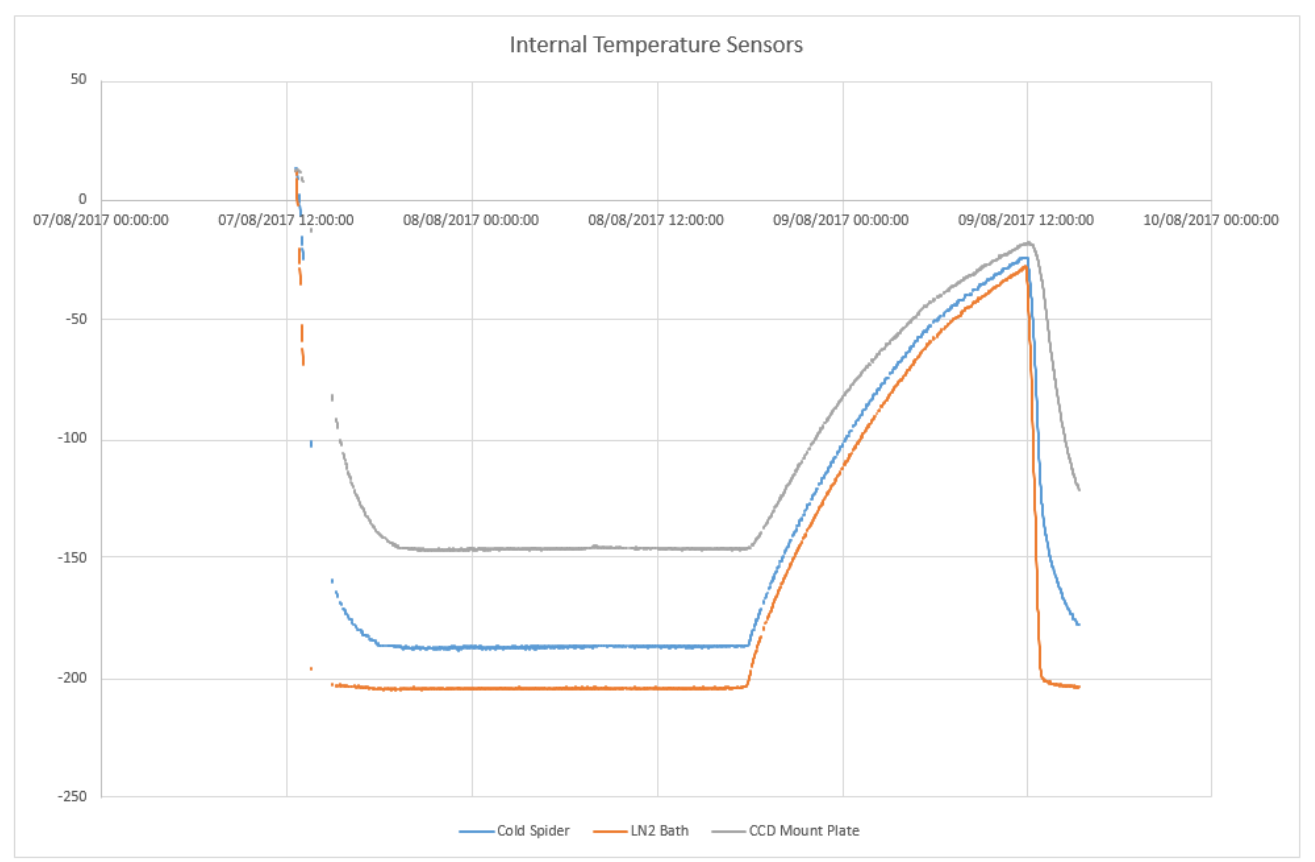

Figure 22 Temperature plot from one liquid nitrogen fill, the $\mathbf{x}$ axis is time in 12 hour increments and the $Y$ axis is degree Celsius. Orange denotes the liquid nitrogen bath temperature, blue - the copper cold spider attached to the back of the CCD mount and grey is the CCD mount plate

\subsection{CCD image area translation in $\mathrm{X}$ and $\mathrm{Y}$}

Both CCDs were imaged periodically (Figure 23) and the results whilst at operating temperature and at ambient were compared.

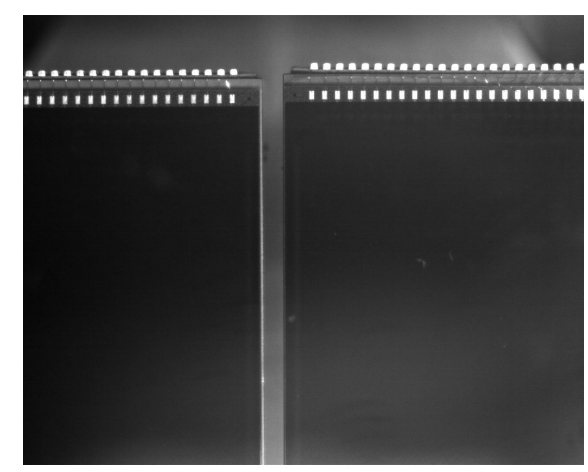

Figure 23 Example Image of CCD positions taken to determine $\mathrm{X}$ and $\mathrm{Y}$ positions of each device.

Table 1 shows the pixel position, as viewed with the Thorlabs CMOS camera, for the top left corner of the right CCD and the top right corner of the left CCD. The pixel scale for these images are 1 pixel equates to $17 \mu \mathrm{m}$ on the CCD. The 
maximum change is 10 pixels, this is when warmed from $150 \mathrm{~K}$ to ambient and this equates to $170 \mu \mathrm{m}$. However the maximum non-repeatability after thermal cycling, at operating temperature is 2 pixels, equating to $34 \mu \mathrm{m}$. This can be calibrated in operation by arc and flat field exposures.

Table 1 translational change due to temperature

\begin{tabular}{|l|l|l|l|l|l|l|l|}
\hline Date & Time & Temp & CCD & X Pos & YPos & Delta X & Delta Y \\
\hline $06 / 10 / 2017$ & $15: 00$ & At temp & Left & 546 & 144 & & \\
\hline $06 / 10 / 2017$ & $15: 00$ & At temp & Right & 594 & 133 & & \\
\hline $09 / 10 / 2017$ & $12: 10$ & Ambient & Left & 541 & 136 & -5 & -8 \\
\hline $09 / 10 / 2017$ & $12: 10$ & Ambient & Right & 597 & 127 & +3 & -6 \\
\hline $09 / 10 / 2017$ & $19: 20$ & At temp & Left & 547 & 142 & +6 & +6 \\
\hline $09 / 10 / 2017$ & $19: 20$ & At temp & Right & 592 & 132 & -5 & +5 \\
\hline $12 / 10 / 2017$ & $08: 00$ & At ambient & Left & 541 & 137 & -6 & -5 \\
\hline $12 / 10 / 2017$ & $08: 00$ & At ambient & Right & 596 & 128 & +4 & -4 \\
\hline $16 / 10 / 2017$ & $19: 20$ & At temp & Left & 547 & 144 & +6 & +7 \\
\hline $16 / 10 / 2017$ & $19: 20$ & At temp & Right & 594 & 133 & -2 & +5 \\
\hline $03 / 11 / 2017$ & $07: 40$ & At ambient & Left & 541 & 134 & -6 & -10 \\
\hline $03 / 10 / 2017$ & $07: 40$ & At ambient & Right & 595 & 126 & +1 & -7 \\
\hline
\end{tabular}




\section{CONCLUSIONS}

Our results show the CCD mount achieves its aim in positioning both CCDs accurately, within specification with sufficient thermal insulation to achieve the desired operating temperature and hold this temperature for beyond the required 15 hours between liquid nitrogen refills.

During vacuum and cool down, the CCDs do not exhibit any signs of deformation further than measured whilst at ambient temperature.

The image plane is repeatable within the dimensional requirements in tip, tilt and piston, and any vibration from the liquid nitrogen boil off is not seen to any significant degree at the CCD surfaces. There was a slow image plane movement of $6 \mu \mathrm{m}$ over the period of test; this is thought to be due to the laboratory ambient temperature change during the night. The specification for ambient temperature within GHRIL is +13 to $+20^{\circ} \mathrm{C}$, similar to the room temperature variations. At least a proportion of this $6 \mu \mathrm{m}$ position change can be attributed to changes of temperature within the confocal measuring head itself. We are therefore not concerned by this small drift which is in any case within specification.

The image areas in $\mathrm{X}$ and $\mathrm{Y}$ are repeatable to within 2 pixels, $34 \mu \mathrm{m}$ between thermal cycles of the CCD assembly. It is envisaged, the spectrograph will be re-calibrated at the start of each night, so this effect can be compensated. However once at temperature we have only seen sub pixel $(<17 \mu \mathrm{m})$ movement. We will carry out further tests regarding image area stability in $\mathrm{X}$ and $\mathrm{Y}$ to confirm we do not see any 'creep' in CCD position over a longer period of time. If this is detected, we propose to constrain one of the CCDs with respect to the other via springs in tension. This will ensure any movement is in one direction only.

\section{REFERENCES}

[1] Rogers, K. et al, "The design of the WEAVE spectrograph", Proceedings of the SPIE, Volume 9147, id. 91476H 15 pp. (2014)

[2] Dalton, G., "WEAVE: The Next Generation Spectroscopy Facility for the WHT", Proceedings of a conference held at Teatro Circo de Marte, Santa Cruz de La Palma, Canary Islands, Spain, p.97, (2016)

[3] Dalton, G. et al, "Final design and progress of WEAVE: the next generation wide-field spectroscopy facility for the William Herschel Telescope", Proceedings of the SPIE, Volume 9908, id. $99081 \mathrm{G} 10$ pp. (2016)

[4] Leuenberger, H., Pearson R.A, "Complication of Radiation Shape Factors for Cylindrical Assemblies", ASME Annual Meeting (1957) (available from http://www.dtic.mil/dtic/tr/fulltext/u2/a284447.pdf) [Accessed $11^{\text {th }}$ May 2018] [5] Engineering ToolBox, "Emissivity Coefficients of some common Materials" (2003) [online] Available at: https://www.engineeringtoolbox.com/emissivity-coefficients-d_447.html [Accessed 11th May 2018] 